\title{
Markers associated with heading and aftermath heading in perennial ryegrass full-sib families
}

\author{
Sai Krishna Arojju 1,3, Susanne Barth', Dan Milbourne1, Patrick Conaghan², Janaki Velmurugan', \\ Trevor R. Hodkinson ${ }^{3}$ and Stephen L. Byrne ${ }^{1 *}$
}

\begin{abstract}
Background: Heading and aftermath heading are important traits in perennial ryegrass because they impact forage quality. So far, genome-wide association analyses in this major forage species have only identified a small number of genetic variants associated with heading date that overall explained little of the variation. Some possible reasons include rare alleles with large phenotypic affects, allelic heterogeneity, or insufficient marker density. We established a genome-wide association panel with multiple genotypes from multiple full-sib families. This ensured alleles were present at the frequency needed to have sufficient statistical power to identify associations. We genotyped the panel via partial genome sequencing and performed genome-wide association analyses with multi-year phenotype data collected for heading date, and aftermath heading.
\end{abstract}

Results: Genome wide association using a mixed linear model failed to identify any variants significantly associated with heading date or aftermath heading. Our failure to identify associations for these traits is likely due to the extremely low linkage disequilibrium we observed in this population. However, using single marker analysis within each full-sib family we could identify markers and genomic regions associated with heading and aftermath heading. Using the ryegrass genome we identified putative orthologs of key heading genes, some of which were located in regions of marker-trait associations.

Conclusion: Given the very low levels of $L D$, genome wide association studies in perennial ryegrass populations are going to require very high SNP densities. Single marker analysis within full-sibs enabled us to identify significant marker-trait associations. One of these markers anchored proximal to a putative ortholog of TFL1, homologues of which have been shown to play a key role in continuous heading of some members of the rose family, Rosaceae.

Keywords: Aftermath heading, Flowering, Genome wide association, Heading, Lolium perenne, Perennial ryegrass, Single marker analysis

\section{Background}

Perennial ryegrass (Lolium perenne L.) is an important forage species grown in temperate regions of the world where it underpins the dairy and livestock sectors. This is due to a high palatability and digestibility [1]. It also displays relatively rapid establishment and has long growing seasons with relatively high yields in suitable environments [2]. With $38 \%$ of global land area available for agriculture, $70 \%$ is assigned as pastoral agricultural land

*Correspondence: stephen.byrne@teagasc.ie

${ }^{1}$ Teagasc, Crop Science Department, Oak Park, Carlow, Ireland

Full list of author information is available at the end of the article
[3]. In Europe alone 76 million hectares is used as permanent pasture [4] and in Ireland $80 \%$ agricultural land (3.4 million hectares) is used for pasture, hay and silage where perennial ryegrass is the preferred species [5].

Heading date is a trait that can have a large effect to the use of perennial ryegrass as a forage [6]. Heading date is associated with a reduction in forage quality [7-9]. The stem and inflorescence formation significantly reduces tiller formation and affects the persistency, digestibility and nutritional value [10]. Perennial ryegrass belongs to the same sub-family (Pooideae) as several other impor- 
tant grain cereals such as barley, oats, rye and wheat [11, 12]. Heading in situations outside of seed production is unwanted as it negatively impacts forage quality by increasing the stem to leaf ratio. Extending the vegetative period would greatly enhance its utility as a forage [13, 14]. Aftermath heading is mainly associated with early heading genotypes, and these tend to show lower persistency and perenniality. There has been limited work done on the genetic control of aftermath heading, and only a single quantitative trait locus (QTL) has been mapped onto linkage group (LG) 6 in an experimental mapping population [9].

In perennial ryegrass, heading is mainly controlled by three main pathways, namely the vernalization pathway, the photoperiod pathway and the circadian clock. To date many QTL mapping studies have been carried out in perennial ryegrass and major loci involved in the floral transition have been identified [9, 15-24]. QTL for heading date have been detected on all seven LGs of perennial ryegrass, with analogous regions on LG4 and LG7 being linked with large affect QTL across multiple populations [17]. Although genes underlying some of these QTL have been proposed $[16,17]$ none have been cloned to date.

In addition to within family based QTL analysis, we can also map QTL in populations using genome wide association analysis (GWAS). This offers the benefit of being able to take advantage of historical recombination to more precisely map the QTL region. In the case of a very rapid decay of linkage disequilibrium (LD), the causative quantitative trait nucleotide (QTN) may be elucidated. However, this does necessitate the need for a high marker density. A recent GWAS study of heading date in perennial ryegrass identified markers affecting heading date across 1,000 F2 families [25]. However, the variation explained by the combined marker set was extremely small. LD only extended to very short distances in the study population, and despite using in excess of 0.9 million SNPs the marker density may be insufficient. Alternatively, rare variants affecting the trait may have resulted in low statistical power to identify associations.

Here, we have developed an association mapping population of 360 individuals coming from six full-sib families with contrasting primary heading dates. Multiple individuals from each full-sib family were selected to ensure any allele will be present at a frequency suitable for association analysis. However, the low levels of LD across families restricted GWAS at our marker density, and so we performed single marker analysis within each full-sib family separately. Anchoring markers to the perennial ryegrass GenomeZipper [26, 27] allowed us to identify regions containing clusters of associated markers, some of which co-located with genes having a known involvement in controlling heading and aftermath heading.

\section{Results and discussion}

\section{Phenotypic variation for heading date and aftermath heading}

The 360 genotypes were planted in two replicates at Oak Park, Carlow, Ireland, and were scored for days to heading in both 2014 and 2015. Spaced plants were scored for the number of days from April 1st until three spikes had emerged on a single plant. In all families, days to heading follows a normal statistical distribution. Plants are generally assigned to one of three groups for heading, these are early (head in first half of May), intermediate (head in second half of May), and late (head in first half of June). The full-sib families G15, G16, and G17 were all developed from late heading parents (Table 1) and this is evident in the phenotypic distributions for these families (Fig. 1). Only G11 had an early heading parent, and G12 and G18 involved intermediate heading parents. Scores for heading date were strongly correlated between 2014 and 2015, with a Pearson's product-moment correlation of 0.82 with a $95 \%$ confidence interval of $(0.79,0.84)$. Variance components were calculated using lme4 [28] genotype as a random effect and year as fixed effect. From this, we calculated heritability on a line mean basis to be 0.88 .

Aftermath heading was scored only in September 2015 using a visual assessment on a scale of 1 (no aftermathheading) to 9 (extensive aftermath heading). The Pearson's product-moment correlation between replicates was 0.68 and a $95 \%$ confidence interval of $(0.61,0.73)$. The difference in aftermath heading scores between replicates was not significant $\left(F_{(1,685)}=3.385, M S E=21.522, P=0.07\right)$ at $\alpha=0.05$. The population mean, sd, and median scores for aftermath heading were 2.7, 2.7 and 1, respectively. We only have a single years data for aftermath heading, however, a recent study of $1453 F_{2}$ families of perennial ryegrass determined heritibalities for aftermath heading that were in line with those determined for heading date [29]. The distribution of scores within each full-sib family, we see that one family (G18) has more variation and a higher propensity for aftermath heading. Taking this family in isolation we looked at the association between heading date and aftermath heading. Using aftermath heading as a response variable in linear regression, we can see that

Table 1 Full-sib family structure

\begin{tabular}{llll}
\hline & Parent1 & Parent2 & Crosses \\
\hline G11 & Pastour & Genesis & Late X Early \\
G12 & Solomon & Tyrella & Inter X Late \\
G15 & Profit X Hercules & Jumbo X Tyrone & Late X Late X Late X Late \\
G16 & AberAvon & Twystar & Late X Late \\
G17 & Tyrconnell & Majestic & Late X Late \\
G18 & AberSilo & Shandon & Inter X Inter
\end{tabular}




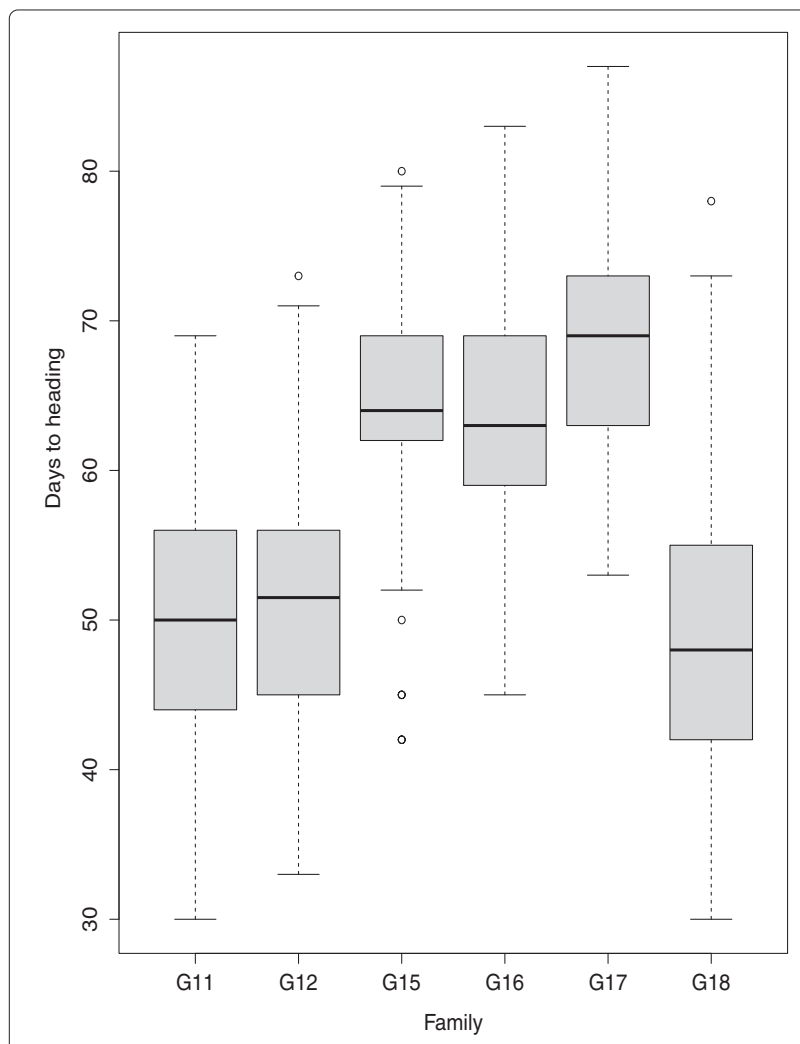

Fig. 1 Phenotypic distribution of heading date in six full-sib families. Boxplots representing heading date in full-sib families with $y$-axis showing days to heading and families on $x$-axis

earlier heading individuals tend to have higher aftermath heading (Additional file 1).

\section{Genome wide association analysis}

We used a genotyping by sequencing approach to characterize variation in the association panel. Data were aligned to the reference genome [30] and variants were identified across the entire 360 genotypes (Table 2). Only variants present in at least $70 \%$ of samples and at a minor allele frequency of $5 \%$ were retained. This left 51,846 SNPs for association analysis with the traits heading date and

Table 2 Markers used at different stages of pipeline

\begin{tabular}{lllll}
\hline Family & $\begin{array}{l}\text { Genotyped } \\
\text { markers }\end{array}$ & $\begin{array}{l}\text { Filtered } \\
\text { markers }\end{array}$ & $\begin{array}{l}\text { Chi-square } \\
\text { threshold }\end{array}$ & $\begin{array}{l}\text { Tagged to } \\
\text { zipper }\end{array}$ \\
All & 51,846 & - & - & - \\
G11 & 27,934 & 15,315 & 7070 & 2174 \\
G12 & 59,524 & 28,606 & 15,564 & 4228 \\
G15 & 77,499 & 32,805 & 19,425 & 4424 \\
G16 & 62,948 & 29,263 & 15,563 & 3421 \\
G17 & 63,516 & 27,007 & 14,315 & 3523 \\
G18 & 17,701 & 6021 & 3075 & 1225 \\
\hline
\end{tabular}

aftermath heading. We corrected for population structure using principal component analysis and the kinship matrix (Fig. 2). The purpose of including 60 genotypes from each of six full-sib families was to inflate the allele frequencies to ensure we had adequate statistical power for association studies. It is possible that many traits in perennial ryegrass may be controlled by rare alleles with large effects, but in order to detect associations an allele must be present in high enough frequency. Within our association panel the rarest allele would, in theory, be present in 30 individuals (each full-sib family is the result of a single pair cross followed by seed multiplication in isolation plots).

We did not find any markers significantly associated with heading date or aftermath heading after correcting for multiple testing (FDR $<0.05$ ). Heading date is a highly heritable trait [29], and one that can be phenotyped very precisely. It was therefore initially surprising that we did not identify any significant associations. Genome wide association studies can fail for many reasons, including a lack of statistical power due to many rare alleles with large effects or allelic heterogeneity. However, to avoid this problem we have used multiple genotypes (60) from each of six full sib families. Another possible explanation is that heading date (and aftermath heading) is highly correlated with population structure, meaning any correction for population structure will result in false negatives. Designing populations to remove any population structure is complicated for a trait like heading date as synchronization of heading is required for cross pollination. Another possible explanation is that the marker density is insufficient to ensure we always have a marker in LD with a QTL. The six $F_{2}$ families were developed from pair-crosses of 12 genotypes taken from a recurrent selection program. When we evaluated the extent of LD in the population, we observed that on average across the genome it decayed very rapidly (Fig. 3). Based on this, our marker density is not sufficient, even considering that our genotyping approach is focused on the non-repetitive and gene-rich fractions of the genome. In this case it is likely that full re-sequencing of the gene space and regions up and downstream is required to capture alleles associated with a trait. A recent GWAS study in perennial ryegrass using almost 20 times the number of markers ( $\sim 1$ million SNPs), did identify significant SNPs for heading. Some SNPs were in close proximity with key heading genes like CONSTANS (CO) and PHYTOCHROME C (PHYC), but the sum of the variances explained by all significant markers was only $20.3 \%$ [25].

We have now established that, in general, marker numbers in the region of 50,000 are going to be unsuitable for GWAS in perennial ryegrass. We believe that our inability to find any significant associations with heading date and aftermath heading was due to low marker density and 

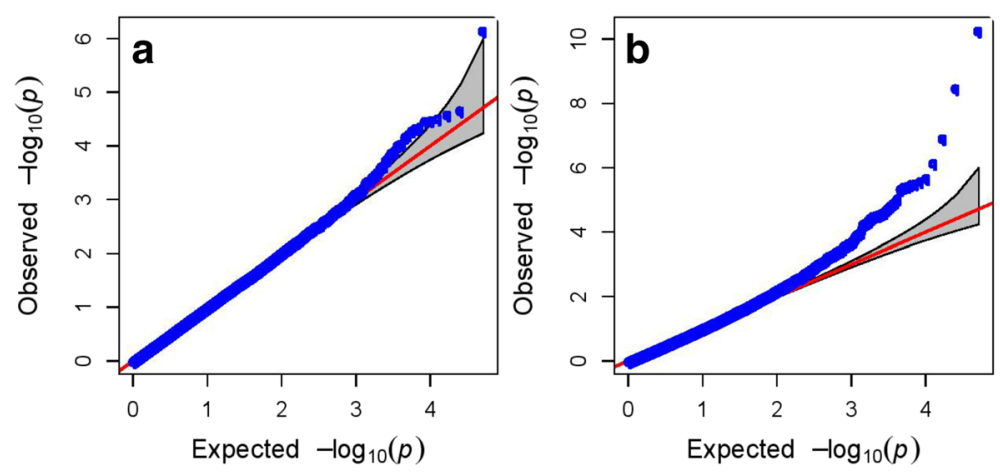

Fig. 2 QQ-plots for $\mathbf{a}$ heading and $\mathbf{b}$ aftermath heading

the extremely low LD in the population. Our population is made up of six full-sib families, and within each family a much higher LD is expected. An alternative approach would be to perform single marker analysis within each full-sib family. There are only 60 genotypes per full-sib family, however, using this approach there is sufficient SNP density to perform a simple marker-trait association analysis within each family separately. This would not enable us to locate the regions directly affecting a phenotype, but would allow us to identify markers linked to QTL.

\section{Single marker analysis in full-sib families}

The original genotypes used in the pair-crosses that generated the six full-sib families were not available for genotyping. We redid the SNP calling on each full-sib family in isolation, and filtered out variants with a minor allele frequency of less than $10 \%$. We only selected SNPs that were segregating in a 1:1 ratio, corresponding to sites that were homozygous in parent one and heterozygous in parent two. This was done because there are only 60 genotypes present in each full-sib family, and so any markers that segregate into more than two marker classes would have a limited number of individuals in each class. A KruskalWallis test was performed on each marker to identify if they were significantly associated with heading date. We then used the perennial ryegrass GenomeZipper [26, 30] to generate a putative order for the markers according to the linkage map upon which the GenomeZipper is based. These data were used to generate heatmaps for each linkage group showing the Kruskal-Wallis test statistic (Figs. 4, 5, 6 and Additional files 2, 3, 4 and 5).

In general the strongest marker-trait associations were identified in the families G11 and G12, particularly on LG4 and LG7 (Figs. 5 and 6). The three families G15, G16, and G17 were all the result of crosses between late heading plants, and these three full-sib families showed the smallest range in days to heading (Fig. 1). Only G11 was from a cross between an early and late heading populations
(Table 1). The PCA shows a separation according to the categorization of parental days to heading on the first principal component, which accounts for $7.8 \%$ of the variation. The two full-sib families involving crosses between parents falling into different heading categories (G11 and G12) are separated from the others on PC1 (Fig. 7). We identified many markers associated with days to heading, particularly on LG4 and LG7 (Figs. 5 and 6). This was not too surprising, considering that many studies in experimental cross-populations have identified large effect QTL on the same linkage groups [9, 15-24].

G18 was the only family that showed a large amount of variation for the aftermath heading. Single marker analysis identified markers significantly associated with aftermath heading anchored onto different LGs using the GenomeZipper (Table 3). In particular we identified markers in five scaffolds anchored to LG6 in a region covering 35.9 to $56.0 \mathrm{cM}$ (Table 3). We also identified markers in two scaffolds on LG2 at 80.4 and $84.2 \mathrm{cM}$, and markers in two scaffolds anchored to LG1 at 31.5 and $31.2 \mathrm{cM}$. The recent release of an annotated draft assembly of the perennial ryegrass genome [30] enables us to identify putative orthologs of key heading genes from model species. Using the GenomeZipper we can locate these on the genetic map and relate them to the marker-trait associations identified above.

\section{Identifying putative orthologs of key heading genes}

Work in model species has identified various genetic pathways controlling heading date (Fig. 8) [31]. Key genes acting within these pathways have also been characterized. We used the perennial ryegrass genome [30] to identify putative perennial ryegrass orthologs to these regulators of heading date. We used protein sequences from Arabidopsis, barley and rice as queries (Table 4) to search the perennial ryegrass protein set, and protein sets from Arabidopsis thaliana [32], Brachypodium distachyon [33], Hordeum vulgare [34], Zea mays [35], Sorghum bicolor [36], and Oryza sativa [37]. Only matches 


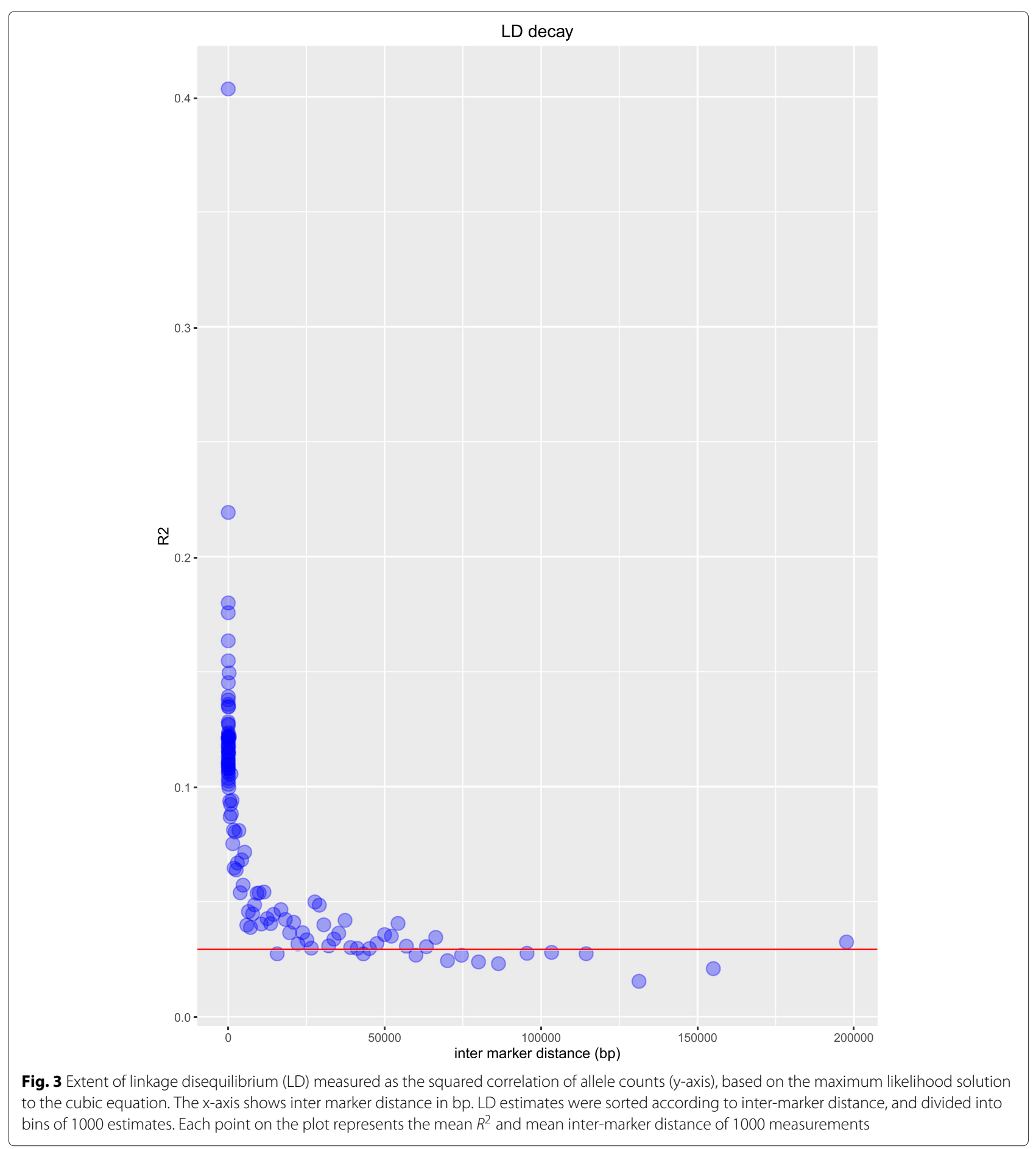

with a minimum query coverage of $60 \%$ and a minimum identity of $50 \%$ were retained for further analysis. The proteins were aligned with an alignment program MUSCLE and phylogenetic trees were built for each of 18 candidate genes. Using phylogenetic trees is the preferred method to establish orthology relationships [38]. Using this approach we were able to identify putative perennial ryegrass orthologs to eleven of these genes (Table 4). We also queried the perennial ryegrass GenomeZipper to identify putative locations for these genes on the genetic map, and relate the locations to markers we anchored as described above.

We identified putative orthologs of the important photo-receptor proteins PHYA (ms_13514|ref0035067- 


\section{LG2}

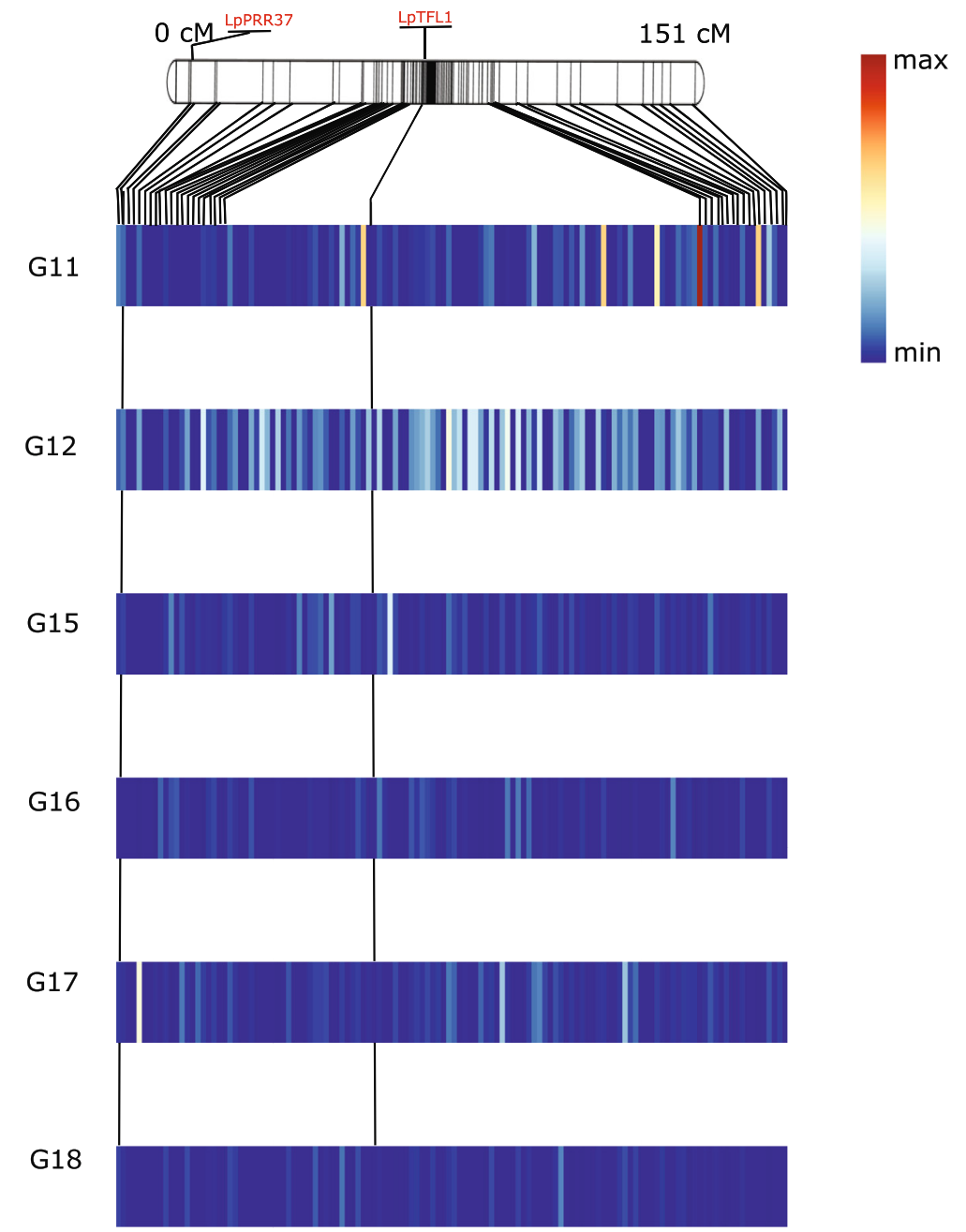

Fig. 4 Heatmap illustrates regions associated with heading over six full-sib families on perennial ryegrass LG2. A Kruskal-Wallis test was performed on each marker to identify significant regions for heading. Using the perennial ryegrass genome zipper [26, 30] we identified a putative gene order for markers on LG2. These data were used to construct the heatmap for each family. A perennial ryegrass transcriptome-based genetic linkage map upon which GenomeZipper was based was used as reference to construct LG2 [26, 27] and placed above the heatmap. Each bar in the heatmap represents region between two genetic markers from the linkage map. The median Kruskal-Wallis test statistic was calculated for markers binned between markers on the genetic linkage map and used to construct the heatmap. Putative orthologs of LpPRR37 and LpTFL1, were identified in the phylogenetic analysis and placed onto LG2 using genetic positions from genome zipper. The genetic positions of these orthologs were extrapolated over the heatmap. Color of the heatmap illustrates the test-statistic of the Kruskal-wallis analysis from 0 to 23

gene-0.0mRNA), PHYB (ms_4484|ref0039062-gene0.0mRNA), PHYC (ms_2801|ref0025790-gene-0.3mRNA) and CRYTOCHROME 2 (CRY2) (ms_4185|ref0010917gene-0.1mRNA) (Fig. 8) (Additional file 6 and 7) and located these on the genetic map via the GenomeZipper (Fig. 5 and Additional file 5). The three Phytochromes, A, B, and C are anchored onto LG4 at different locations. All three are in locations where markers significantly associated with days to heading in one or more full-sib families. CRY2 is located on LG6 at $52.5 \mathrm{cM}$, in a region where we identified a cluster of markers between 35.9 and $56 \mathrm{cM}$ that were associated with aftermath heading in G18 (Table 3). We also identified putative orthologs to PSEUDO RESPONSE REGULATOR PROTEIN 37 (PRR37) (ms_13366|ref0021945-gene-0.0mRNA) and SUPRESSOR OF OVEREXPRESSION OF CO 1 (SOC1) (ms_6002|ref0025562-gene-0.0mRNA) that play important roles in the central circadian clock (Additional file 8 and 9) (Fig. 4). Another important photoperiodic pathway gene is $\mathrm{CO}$, which directly regulates the key 


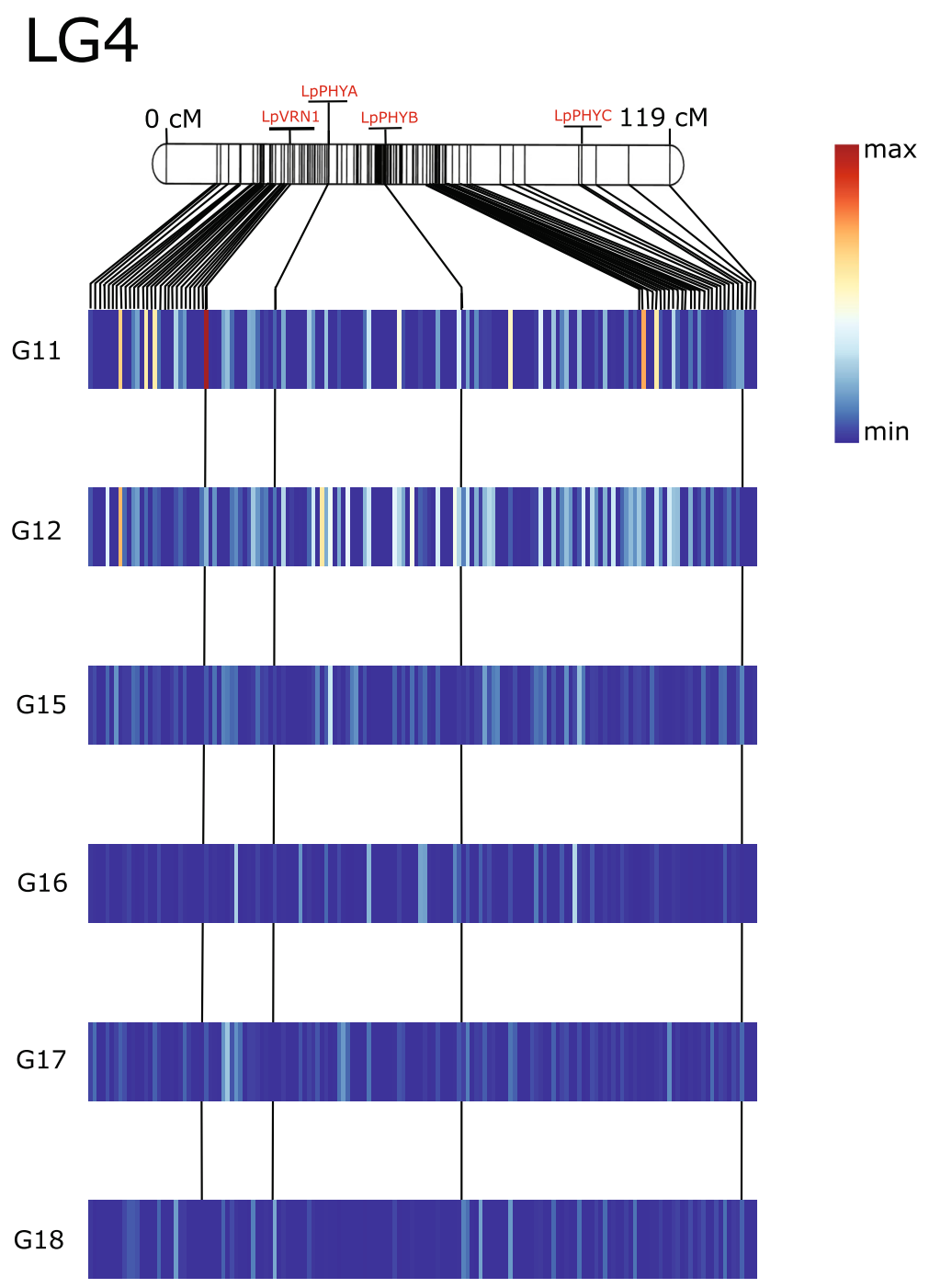

Fig. 5 Heatmap illustrates regions associated with heading over six full-sib families in perennial ryegrass LG4. A Kruskal-Wallis test was done on each marker to identify significant regions for heading. Using perennial ryegrass genome zipper $[26,30]$ putative gene order for markers on LG4 was identified. These data was used to construct the heatmap for each family. Ryegrass transcriptome linkage map upon which GenomeZipper was based is used as reference to construct LG4 $[26,27]$ and placed above the heatmap. Each bar in the heatmap represents region between two genetic markers from the linkage map. The median Kruskal-Wallis test statistic was calculated for bins represented by gaps between markers on the genetic linkage map and used to construct the heatmap. Putative orthologs of LpVRN1, LPPHYA, LPPHYB and LpPHYC, were identified in the phylogenetic analysis and placed onto LG4 using genetic positions from genome zipper. The genetic positions of these orthologs were extrapolated over the heatmap as bars. Color of the heatmap illustrates the test-statistic of the Kruskal-wallis analysis from 0 to 21

floral activator FT (Fig. 8), and we have found a putative ortholog to CO (ms_5059|ref0019898-gene-0.1mRNA) (Additional file 10) in perennial ryegrass that anchored onto LG7 at 43.5cM (Fig. 6).

Both the regulation of $\mathrm{CO}$ and stability of photoreceptors is controlled by GIGANTEA (GI) that is generally believed to be single copy, with a highly conserved role across the angiosperms [39]. We identified a putative ortholog to GI (ms_1276|ref0038679-gene-0.4mRNA) (Additional file 11) in our analysis that anchored onto LG3 at 29.3cM [3] (Additional file 3). A scaffold with markers significantly associated with aftermath heading in G18 was anchored to LG3 at $28.8 \mathrm{cM}$. In addition to genes from the photoperiodic pathway, we identified a putative ortholog of the barley VERNALIZATION 1 (VRN1) (ms_312|ref002704-gene-0.1mRNA) protein that is involved in the vernalization pathway (Additional file 12). The VRN1 protein is anchored on LG4 at $31.4 \mathrm{cM}$ (Fig. 5) in a region with markers significantly associated with days to heading. This was most evident in the full-sib family G11 that was generated from crossing early and late heading populations. 


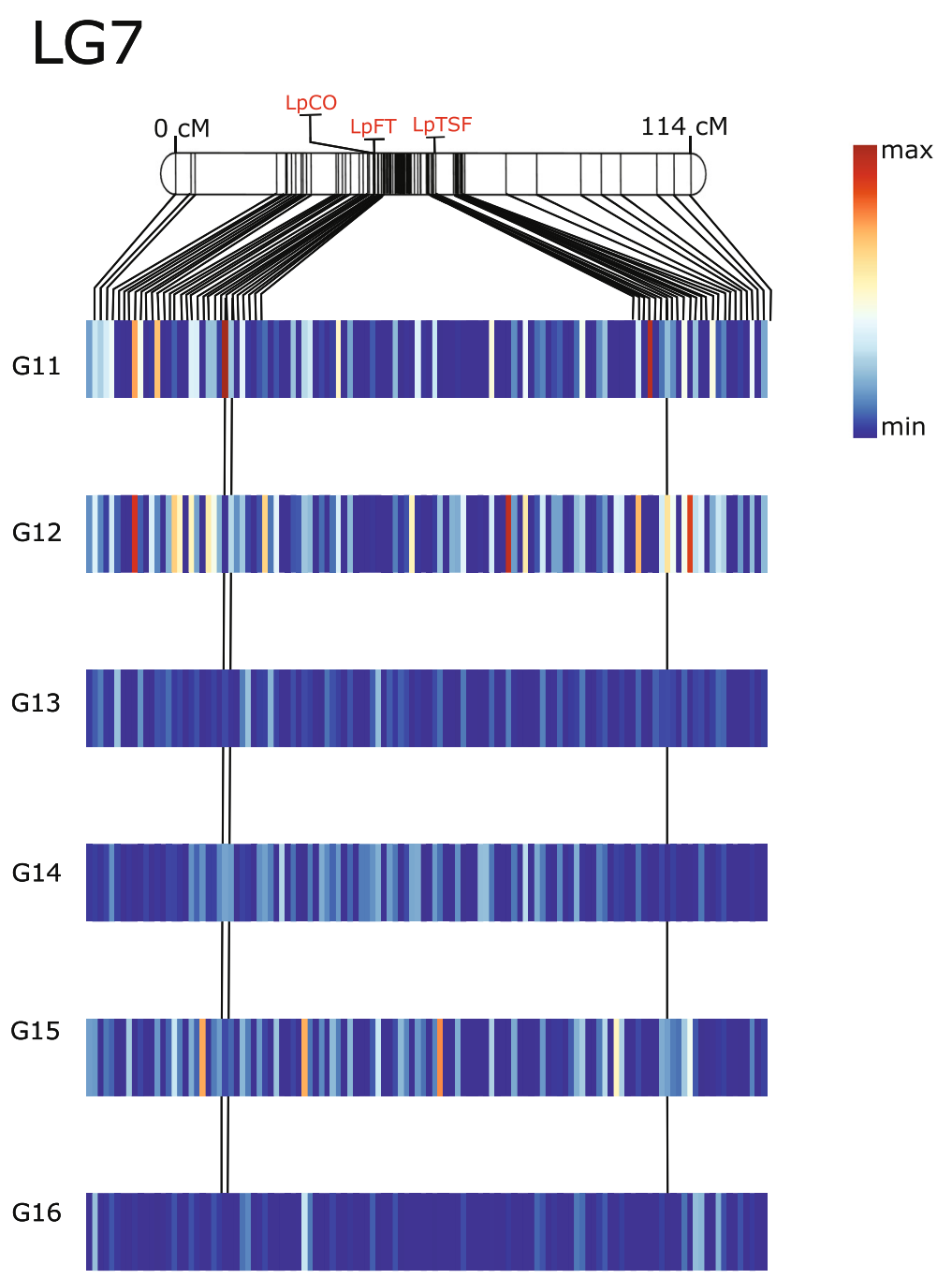

Fig. 6 Heatmap illustrates regions associated with heading over six full-sib families in perennial ryegrass LG7. A Kruskal-Wallis test was done on each marker to identify significant regions for heading. Using perennial ryegrass genome zipper [26, 30] putative gene order for markers on LG7 was identified. These data was used to construct the heatmap for each family. Ryegrass transcriptome linkage map upon which GenomeZipper was based is used as reference to construct LG7 [26,27] and placed above the heatmap. Each bar in the heatmap represent region between two genetic markers from linkage map. The median Kruskal-Wallis test statistic was calculated for bins represented by gaps between markers on the genetic linkage map and used to construct the heatmap. Putative orthologs of LpCO and LpFT, were identified in the phylogenetic analysis and placed onto LG7 using genetic positions from genome zipper. The genetic positions of these orthologs were extrapolated over the heatmap. Color of the heatmap illustrates the test-statistic of the Kruskal-wallis analysis from 0 to 12

It has already been shown that a dominant mutation in VRN1 promoter region is responsible for changes in growth habit of winter wheat to spring wheat [40]. The vernalisation and the photoperiod pathway influence heading by acting on the key floral pathway integrator FT (Fig. 8).

\section{Perennial ryegrass orthologs of $\mathrm{FT}$ and TFL1}

Floral transition is controlled by FLOWERING LOCUS $\mathrm{T}$ (FT) and TERMINAL FLOWERING 1 (TFL1), which are genes that have functionally diverged from a common ancestor MOTHER OF FT AND TFL1 (MFT) [41].
FT promotes heading whereas TFL1 represses heading. In Arabidopsis the FT/TFL1 gene family consists of six members: FT, TFL1, MFT, BROTHER OF FT (BFT), CENTRORADIALIS (CEN), and TWIN SISTER OF FT (TSF). They share high sequence similarity but do have different roles in floral transition [42]. Using the Arabidopsis FT protein as a query we found perennial ryegrass proteins with sequence similarity to FT/TFL1 family proteins (Fig. 9). We also identified similar proteins in Brachypodium and barley. A phylogenetic analysis using the maximum likelihood method divided the proteins into two distinct groups, one group with the floral inducers 


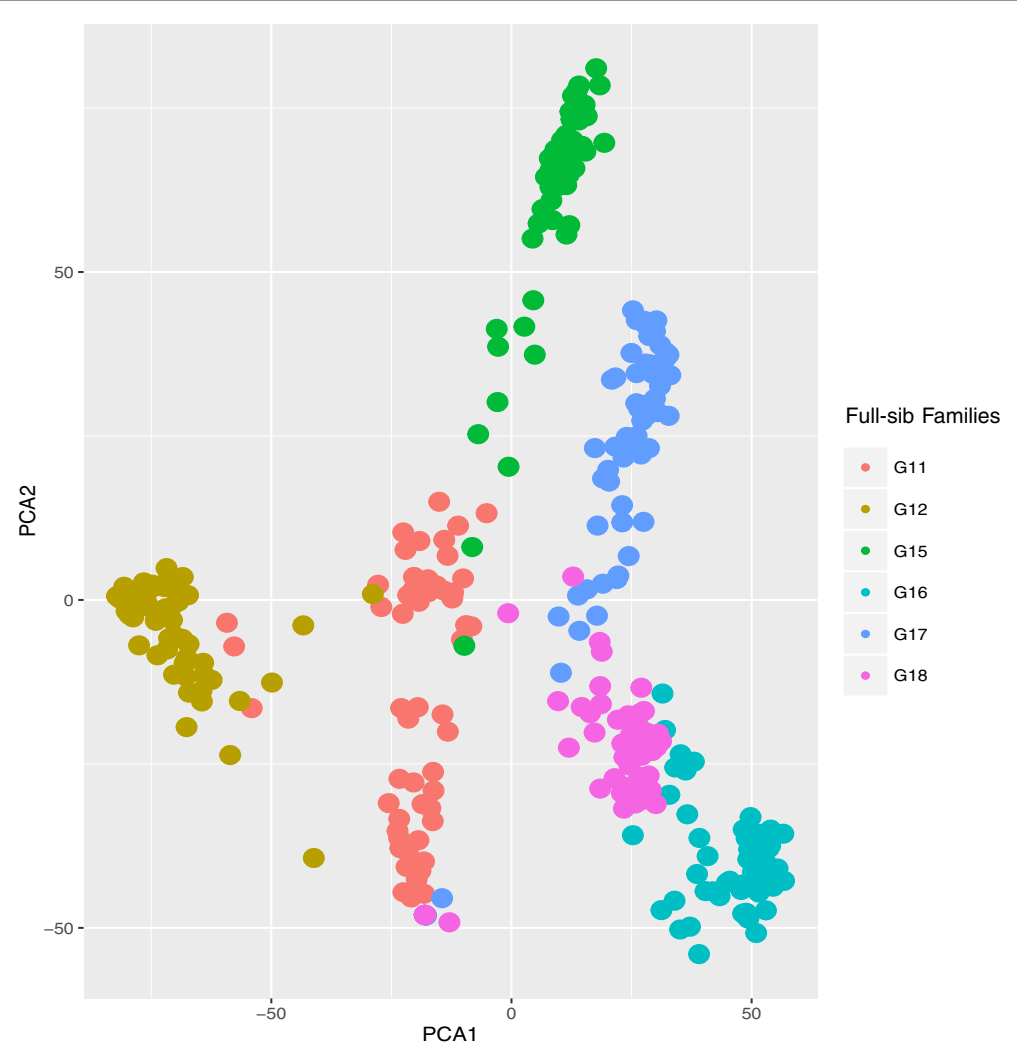

Fig. 7 Principal component analysis (PCA) of 360 perennial ryegrass individuals, genotyped using 51,846 SNPs. The first two principal components explained $14.8 \%$ of total variation. Components are colored according to family (color coding is listed in figure legend)

FT and TSF and another group with the floral inhibitors TFL1, CEN and BFT (Fig. 9) [43].

Apart from floral transition, Arabidopsis FT also mediates stomatal opening [44]. Likewise, TFL1 is also involved in meristematic development and perennial heading [45]. We identified a putative perennial ryegrass ortholog of FT (ms_13332|ref0029013-gene-0.0mRNA) (Fig. 9) that was anchored to LG7 at $43.6 \mathrm{cM}$, in a region with markers significantly associated with heading (Fig. 6). FT was anchored to the same genetic position $(43.6 \mathrm{cM})$, as a previously mapped genetic marker, LpVRN3 [26]. LpVRN3 was designed on a sequence that shared $100 \%$ identity (alignment length of $80.4 \%$ ) with the transcript we identified as orthologus to FT. Two genes from the FT/TFL gene family have previously been mapped in perennial ryegrass [27]. Both were mapped in the same experimental population used as the backbone to the GenomeZipper. Using the available genome data we can now better identify the putative perennial ryegrass orthologs to these genes. Based on our phylogentic anlaysis, the genetic marker previously labeled as LpFT is more likely to be an ortholog of TSF (ms_9269|ref0005840-gene-0.0mRNA) (Fig. 9). TSF is the closet sequence homologue of FT and they have overlapping roles in promoting heading, however it does have a distinct role to play under short day conditions [46]. TSF was anchored to LG7 at 57.2cM (Fig. 6) in a region with markers significantly associated with heading date, particularly in family G11.

The Arabidopsis floral inhibitors, TFL1, BFT and CEN were grouped in a branch separate to FT. We identified putative perennial ryegrass orthologs of TFL1 (ms_821|ref0016245-gene-0.0mRNA) clustering with barley TFL1 (Fig. 9) that was anchored on the GenomeZipper to LG2 at $79.8 \mathrm{cM}$ (Fig. 4). Previously a perennial ryegrass gene with sequence homology to TFL1 was anchored to LG5 at 27.5cM using a transcriptome based genetic map [27], however, our phylogentic analysis suggests that this is more likely an ortholog of BFT. In Arabidopsis BFT shares highest sequence similarity to TFL1 and functions similar to TFL1 in meristematic development to repress heading [47]. Interestingly, on LG2 markers we identified in single marker analysis for aftermath heading, were located on the GenomeZipper at $80.8 \mathrm{cM}$ and $84.2 \mathrm{cM}$. These markers were next to putative perennial ryegrass ortholog of TFL1.

In perennial ryegrass, TFL1 is characterized as a repressor of heading and a regulator of axillary meristem identity [48]. When LpTFL1 was overexpressed in 
Table 3 Single marker analysis for aftermath heading

\begin{tabular}{|c|c|c|c|c|c|c|}
\hline Scaffold & Position & Test statistic & $p$-value & $q$-value & LG & $\mathrm{CM}$ \\
\hline 7674 & 27953 & 16.05 & $6.15 \times 10^{-05}$ & 0.045 & 1 & 57.5 \\
\hline 7094 & 37030 & 15.46 & $8.39 \times 10^{-05}$ & 0.045 & 3 & 60.4 \\
\hline 32847 & 126 & 14.82 & 0.00011 & 0.045 & - & - \\
\hline 9166 & 29048 & 14.26 & 0.00015 & 0.045 & 3 & 28.8 \\
\hline 4946 & 43369 & 13.69 & 0.00021 & 0.045 & 4 & 0 \\
\hline 4946 & 43375 & 13.69 & 0.00021 & 0.045 & 4 & 0 \\
\hline 9706 & 5583 & 13.24 & 0.00027 & 0.045 & - & - \\
\hline 444 & 103726 & 13.08 & 0.00029 & 0.045 & 2 & 84.2 \\
\hline 3234 & 46150 & 12.09 & 0.00032 & 0.045 & - & - \\
\hline 444 & 103736 & 12.69 & 0.00036 & 0.045 & 2 & 84.2 \\
\hline 444 & 103741 & 12.69 & 0.00036 & 0.045 & 2 & 84.2 \\
\hline 13778 & 13753 & 12.69 & 0.00036 & 0.045 & - & - \\
\hline 14987 & 7361 & 12.56 & 0.00039 & 0.045 & - & - \\
\hline 8926 & 31874 & 12.55 & 0.00039 & 0.045 & 6 & 40.8 \\
\hline 8309 & 12264 & 12.51 & 0.00040 & 0.045 & 1 & 31.5 \\
\hline 8309 & 12267 & 12.51 & 0.00040 & 0.045 & 1 & 31.5 \\
\hline 4418 & 6989 & 12.43 & 0.00042 & 0.045 & 1 & 31.2 \\
\hline 1397 & 62074 & 12.41 & 0.00042 & 0.045 & 4 & 34.0 \\
\hline 4165 & 42625 & 12.31 & 0.00044 & 0.045 & 6 & 55.2 \\
\hline 9166 & 29013 & 12.23 & 0.00046 & 0.045 & 3 & 28.8 \\
\hline 9166 & 29061 & 12.23 & 0.00045 & 0.045 & 3 & 28.8 \\
\hline 4418 & 6933 & 12.18 & 0.00048 & 0.045 & 1 & 31.2 \\
\hline 8672 & 34875 & 12.11 & 0.00050 & 0.045 & - & - \\
\hline 16758 & 8509 & 12.03 & 0.00052 & 0.045 & 5 & 26.4 \\
\hline 9159 & 27424 & 11.91 & 0.00055 & 0.045 & 6 & 44.5 \\
\hline 500 & 65053 & 11.63 & 0.00064 & 0.045 & 6 & 35.9 \\
\hline 1264 & 86342 & 11.63 & 0.00064 & 0.045 & - & - \\
\hline 444 & 103718 & 11.58 & 0.00066 & 0.045 & 2 & 84.2 \\
\hline 444 & 103742 & 11.58 & 0.00066 & 0.045 & 2 & 84.2 \\
\hline 19325 & 5681 & 11.52 & 0.00068 & 0.045 & 1 & 13.0 \\
\hline 5444 & 49105 & 11.51 & 0.00068 & 0.045 & 1 & 17.2 \\
\hline 498 & 2811 & 11.51 & 0.00069 & 0.045 & 3 & 41.8 \\
\hline 498 & 2853 & 11.51 & 0.00069 & 0.045 & 3 & 41.8 \\
\hline 5379 & 10675 & 11.41 & 0.00072 & 0.046 & 3 & 28.8 \\
\hline 4265 & 21824 & 11.33 & 0.00076 & 0.047 & 6 & 56.0 \\
\hline 14987 & 7423 & 11.15 & 0.00084 & 0.048 & 4 & 45.3 \\
\hline 9529 & 16294 & 11.11 & 0.00085 & 0.048 & 1 & 13.4 \\
\hline 1180 & 59276 & 11.08 & 0.00087 & 0.048 & 2 & 80.4 \\
\hline 6377 & 12180 & 11.07 & 0.00087 & 0.048 & 1 & 17.2 \\
\hline 44 & 244288 & 11.02 & 0.00089 & 0.048 & 4 & 66.1 \\
\hline 130 & 8254 & 10.83 & 0.00099 & 0.050 & 4 & 79.01 \\
\hline 898 & 54740 & 10.80 & 0.0010 & 0.050 & 3 & 50.3 \\
\hline 898 & 54774 & 10.80 & 0.0010 & 0.050 & 3 & 50.3 \\
\hline 444 & 103617 & 10.76 & 0.0010 & 0.050 & 2 & 84.2 \\
\hline 444 & 103552 & 10.69 & 0.0010 & 0.051 & 2 & 84.2 \\
\hline 762 & 70380 & 10.67 & 0.0010 & 0.051 & 3 & 40.8 \\
\hline 4165 & 18375 & 10.49 & 0.0011 & 0.051 & 6 & 55.2 \\
\hline 441 & 136238 & 10.42 & 0.0012 & 0.051 & 4 & 45.3 \\
\hline 441 & 136239 & 10.49 & 0.0012 & 0.051 & 4 & 45.3 \\
\hline 9861 & 5433 & 10.40 & 0.0012 & 0.051 & 4 & 27.9 \\
\hline
\end{tabular}

Arabidopsis, plants displayed a delayed heading phenotype and extended vegetative growth [48]. In perennial ryegrass expression level of LPTFL1 was observed in leaves, inflorescence, roots, stem and apex. It was found that after a period of cold (primary induction), expression levels of LpTFL1 reduced, allowing plants to prepare for heading. As the day length and temperature increases (secondary induction), LpTFL1 is upregulated in the apex to promote tillering [48]. Unlike annual grasses that flower once in the season and die after seed production, perennial ryegrass continues to grow even after seed production by maintaining at least one tiller in a vegetative phase. It was shown that tillering in ryegrass is mainly controlled by spatiotemporal regulatory mechanism, by activating certain genes to repress heading in vernalized tillers [49]. Interestingly, mutations in homologues of TFL1 in rose $(R o K S N)$ and woodland strawberry (FvKSN) (both Rosoideae members of the rose family Rosaceae) have been shown to be responsible for continuous heading phenotypes in these species [50]. The putative ortholog of TFL1 identified here, which co-locates with variants for aftermath heading, is an interesting candidate gene for further study of this important forage quality trait.

\section{Conclusions}

In this study we did not detect any SNPs significantly associated with heading and aftermath heading in a genomewide association analysis, most likely due to the rapid decay of LD we observed in the population. However, using single marker analysis within each full-sib family we did identify linked markers, some in regions containing putative orthologs of key heading genes. Interestingly, in a family segregating for aftermath heading, SNPs were anchored proximal to a putative ortholog of TFL1, homologues of which have recently been shown to play a key role in continuous heading/aftermath heading of some Rosaceae species [50].

\section{Methods}

\section{Plant material and phenotypic data}

The association population consisted of 360 individual plants from six full-sib F2 families created at Oak Park, Carlow, Ireland (60 individuals selected at random from each family) (Table 1). The parents used to create the fullsib families originated from perennial ryegrass varieties (Table 1). Plants were established in the glasshouse and then transplanted into the field in a spaced plant nursery in 2013 at Oak Park, Carlow, Ireland in two replicates. Each replicate consisted of 30 blocks with 2 individuals from each full-sib family within a block. The number of days to heading from April $1^{\text {st }}$ was monitored in 2014 and 2015 for each plant. An individual plant was considered as headed, when three or more heads had emerged from the leaf sheath. In the same population aftermath heading 


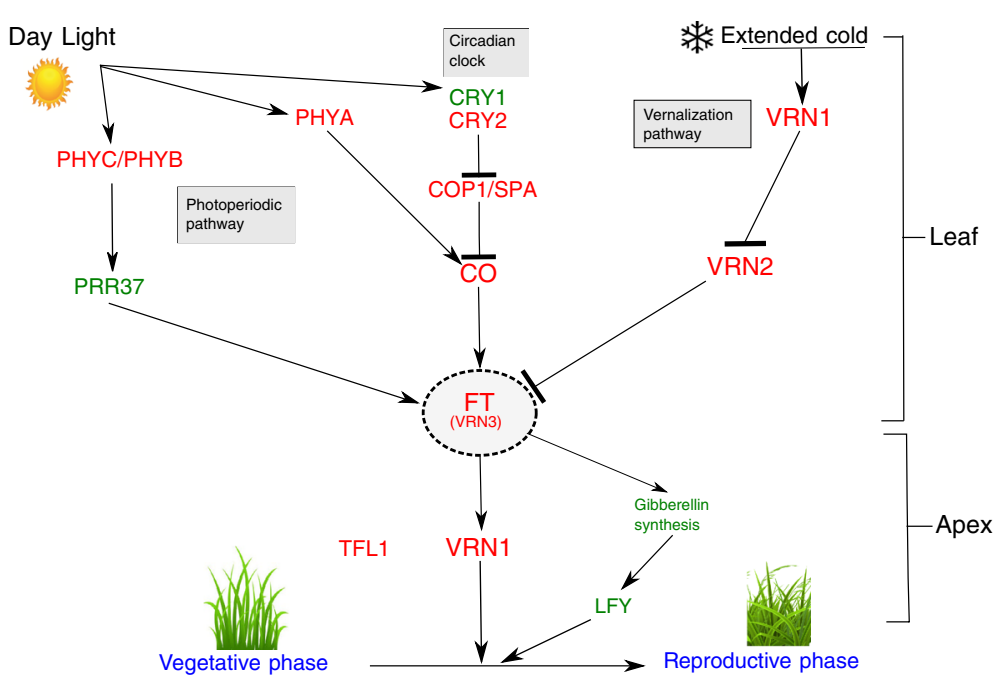

Fig. 8 Schematic view of genetic pathway controlling heading. Genes promoting heading were shown by arrows and genes acting as repressor shown as lines with bars. External factors like day light and extended cold periods were represented with respective symbols. Pathways were mentioned in grey boxes and genes shown in red were considered as key regulators in heading

was visually scored in the year 2015 on a scale of 1 (no aftermath heading) to 9 (intense aftermath heading) as described in [25]. Using the $\mathrm{R}$ package lme4 [28] variance components were estimated for heading date using genotype as a random effect and year as fixed effect. Conditional means were calculated and used for subsequent analysis.

\section{Genotyping full-sib families}

We used a genotyping-by-sequencing approach that followed the protocol developed by Elshire et al. [51]. Briefly, genomic DNA was isolated from each individual, digested with ApeKI, samples were grouped into libraries, amplified, and sequenced on an Illumina HiSeq 2000. After sequencing, adaptor contamination was removed with Scythe [52] with a prior contamination rate set to
0.40. Sickle [53] was used to trim reads when the average quality score in a sliding window (of $20 \mathrm{bp}$ ) fell below a phred score of 20 , and reads shorter than 40bp were discarded. The reads were demultiplexed using sabre [54] and data from each sample was aligned to the perennial ryegrass reference genome [30] using BWA [55]. The Genome Analysis Tool Kit (GATK) [56] was used to identify putative variants across the full-sib families, and also within each full-sib family. Only genotype calls with a phred score of 30 (GQ, Genotype Quality), and only variant sites with a mean mapping quality of 30 were retained. In the case of the SNP set across all full-sib families, we used a minimum minor allele frequency threshold of $5 \%$ (Additional file 13). When identifying an SNP set within each full-sib family we used a minimum minor allele frequency threshold of $10 \%$.

Table 4 Phylogenetic relationships of candidate genes involved in heading

\begin{tabular}{llllll}
\hline Query candidate & Species & Protein near query & Position on Zipper & Genetic position on Zipper & Phylogenetic tree \\
\hline TFL1 & Arabidopsis & ms_821|ref0016245 & LG2-79.8cM & LG5-27.5cM & Figure 9 \\
FT & Arabidopsis & ms_13332|ref0029013 & LG7-43.6cM & LG7-57.3cM & Figure 9 \\
CRY2 & Arabidopsis & ms_4185|ref0010917 & LG6-52.5cM & LG6-52.5cM & Additional file 7 \\
Gl & Arabidopsis & ms_1276|ref0038679 & LG3-29.6cM & NA & Additional file 11 \\
PHYA & Arabidopsis & ms_13514|ref0035067 & LG4-38.5cM & LG4-38.5cM & Additional file 6 \\
PHYB & Arabidopsis & ms_4484|ref0039062 & LG4-51.0cM & LG4-51.0cM & Additional file 6 \\
PHYC & Arabidopsis & ms_2801|ref0025790 & LG4-98.2cM & LG4-98.2CM & Additional file 6 \\
PRR37 & Rice & ms_13366|ref0021945 & LG2-12.4CM & NA & Additional file 8 \\
SOC1 & Arabidopsis & ms_6002|ref0025562 & LG6-0CM & NA & Additional file 9 \\
VRN1 & Barley & ms_312|ref0002704 & LG4-31.4CM & LG4-31.4Cm & Additional file 12 \\
CO & Rice & ms_5059|ref001989 & LG7-43.5cM & LG7-42.7cM & Additional file 10 \\
\hline
\end{tabular}




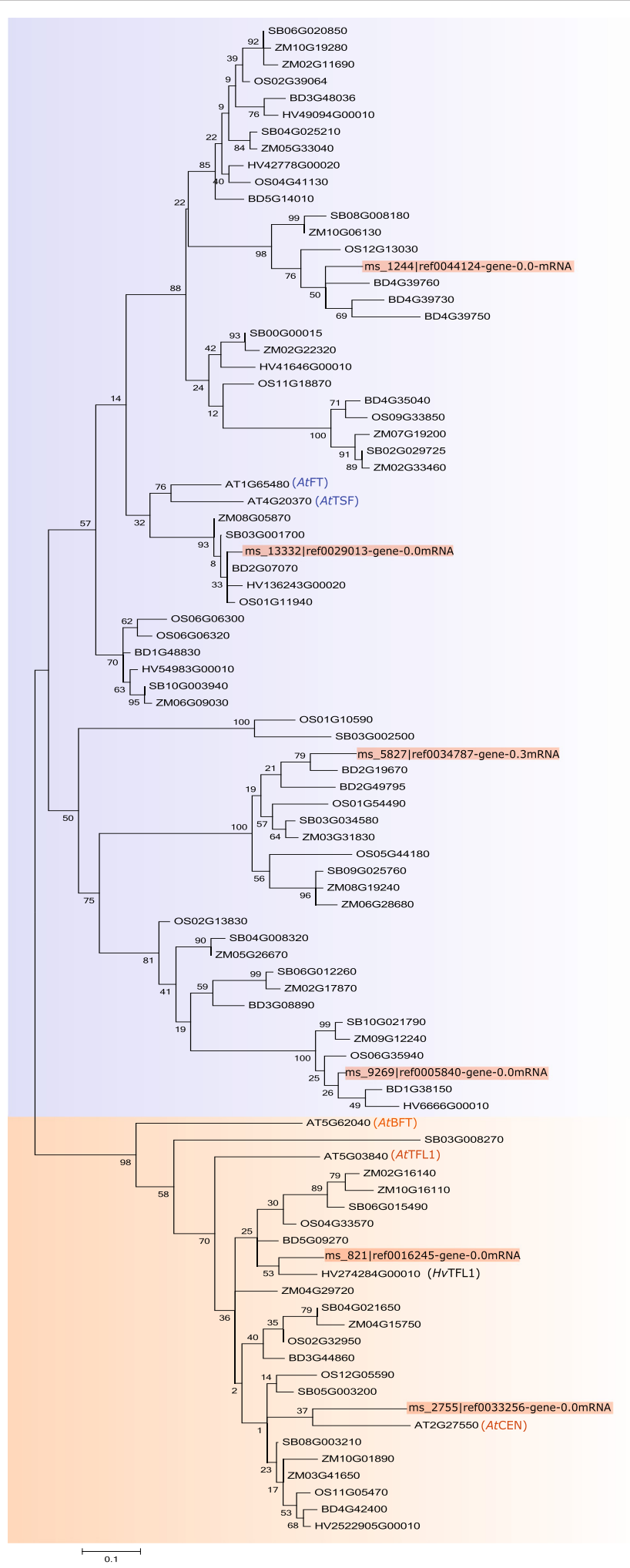

Fig. 9 Phylogenetic analysis of FT/TFL1 gene family using Arabidopsis FT as query. The evolutionary history was inferred by using the Maximum Likelihood method based on the JTT matrix-based model [67]. Bootstrap values after 100 replicates were shown next to the branches. The tree is mid-point rooted, drawn to scale, with branch lengths proportional to the number of substitutions per site. The analysis involved 90 amino acid sequences. All positions containing gaps and missing data were eliminated. There were a total of 83 positions in the final dataset. Evolutionary analyses were conducted in MEGA 6.06 [66]. All the associated Lolium proteins are in red and Arabidopsis proteins were highlighted 


\section{Genome wide association and linkage disequilibrium (LD) analysis}

A mixed linear model implemented in the $\mathrm{R}$ package GAPIT (Genomic Association and Prediction Integrated Tool) [57] was used to perform an association analysis. The mixed model accounts for population structure and family relatedness using principal component analysis (PCA) and a kinship matrix calculated by GAPIT with available input genotypic data. To account for multiple testing during association analysis, false discovery rate (FDR) [58] and Bonferroni correction (0.05/51864) [59] with an $\alpha$ level of 0.05 was used setting a threshold at $9.8 \times 10^{-7}$. To assess the extent of LD across the fullsib populations we identified SNPs located within a single genomic scaffold, and calculated the inter SNP distance and the squared correlation of the allele counts in Plink 1.9 [60], based on the maximum likelihood solution to the cubic Eq. [61].

\section{Pipeline for single marker analysis}

A SNP panel was developed for each full-sib family, using a $10 \%$ of minor allele frequency, and subsequent analyis was performed on each of the six full sib families independently. SNPs segregating in a 1:1 ratio were selected, that is homozygous in parent one and heterozygous in parent two. A $X^{2}$ test was used to eliminate SNPs that deviated significantly from a 1:1 segregation. We then performed a Kruskal-Wallis test using R [62] on each marker to check for association with heading date. Using the GenomeZipper [26, 30] we established a putative order for these markers along the genetic map. The median Kruskal-Wallis test statistic was calculated for bins represented by gaps between markers on the genetic linkage map.

\section{Protein datasets and phylogenetic analysis}

The query proteins for key heading genes was obtained from Arabidopsis thaliana, rice and barley using the uniport database [63] (Table 4). The complete protein sets from perennial ryegrass [30], Arabidopsis [32], Brachypodium [33], barley [34], rice [37], Sorghum [36] and maize [35] were gathered from PLAZA 3.0 [64] and combined into single file to build a BLASTp database. Using each query we performed a BLASTp with an evalue of 10e10 and parsed the results for hits with at least $60 \%$ coverage and $50 \%$ identity. The sequences were aligned using MUSCLE [65], an alignment program implemented in MEGA 6.06 [66]. The phylogentic analysis was carried out using the Maximum Likelihood method based on the JTT matrix-based model in MEGA 6.06 [66, 67]. Bootstrap values after 100 replicates are shown next to the branches. Initial tree(s) for the heuristic search were obtained automatically by applying Neighbor-Join and BioNJ algorithms to a matrix of pairwise distances estimated using a JTT model, and then selecting the topology with superior log likelihood value. The tree is mid-point rooted, drawn to scale, with branch lengths measured in the number of substitutions per site.

\section{Additional files}

Additional file 1: Figure S1. Scatter plot for heading vs aftermath heading. Linear regression was done using aftermath heading as response variable and heading as explantory variable for family G18. Early heading genotypes showed tendency of higher aftermath heading. (PDF $10 \mathrm{~kb}$ )

Additional file 2: Figure S2. Heatmap of perennial ryegrass LG1 over six full-sib families. A Kruskal-Wallis test was performed on each marker to identify significant regions for heading. Using the perennial ryegrass genome zipper $[26,30]$ we identified a putative gene order for markers on LG1. These data were used to construct the heatmap for each family. A perennial ryegrass transcriptome-based genetic linkage map upon which GenomeZipper was based was used as reference to construct LG1 $[26,27]$ and placed above the heatmap. Each bar in the heatmap represents region between two genetic markers from the linkage map. The median Kruskal-Wallis test statistic was calculated for markers binned between markers on the genetic linkage map and used to construct the heatmap. Color of the heatmap illustrates the test-statistic of the Kruskal-wallis analysis. (PDF $31 \mathrm{~kb}$ )

Additional file 3: Figure S3. Heatmap of perennial ryegrass LG3 over six full-sib families. A Kruskal-Wallis test was performed on each marker to identify significant regions for heading. Using the perennial ryegrass genome zipper $[26,30]$ we identified a putative gene order for markers on LG3. These data were used to construct the heatmap for each family. A perennial ryegrass transcriptome-based genetic linkage map upon which GenomeZipper was based was used as reference to construct LG3 [26, 27] and placed above the heatmap. Each bar in the heatmap represents region between two genetic markers from the linkage map. The median Kruskal-Wallis test statistic was calculated for markers binned between markers on the genetic linkage map and used to construct the heatmap. Putative ortholog of LpGl, was identified in the phylogenetic analysis and placed onto LG3 using genetic positions from genome zipper. The genetic positions of these orthologs were extrapolated over the heatmap. Color of the heatmap illustrates the test-statistic of the Kruskal-wallis analysis. (PDF $26 \mathrm{~kb}$ )

Additional file 4: Figure S4. Heatmap of perennial ryegrass LG5 over six full-sib families. A Kruskal-Wallis test was performed on each marker to identify significant regions for heading. Using the perennial ryegrass genome zipper $[26,30]$ we identified a putative gene order for markers on LG5. These data were used to construct the heatmap for each family. A perennial ryegrass transcriptome-based genetic linkage map upon which GenomeZipper was based was used as reference to construct LG5 [26, 27] and placed above the heatmap. Each bar in the heatmap represents region between two genetic markers from the linkage map. The median Kruskal-Wallis test statistic was calculated for markers binned between markers on the genetic linkage map and used to construct the heatmap. Color of the heatmap illustrates the test-statistic of the Kruskal-wallis analysis. (PDF $32 \mathrm{~kb}$ )

Additional file 5: Figure S5. Heatmap of perennial ryegrass LG6 over six full-sib families. A Kruskal-Wallis test was performed on each marker to identify significant regions for heading. Using the perennial ryegrass genome zipper $[26,30]$ we identified a putative gene order for markers on LG6. These data were used to construct the heatmap for each family. A perennial ryegrass transcriptome-based genetic linkage map upon which GenomeZipper was based was used as reference to construct LG6 [26, 27] and placed above the heatmap. Each bar in the heatmap represents region between two genetic markers from the linkage map. The median Kruskal-Wallis test statistic was calculated for markers binned between markers on the genetic linkage map and used to construct the heatmap. Putative ortholog of LPCRY2 was identified in the phylogenetic analysis and placed onto LG6 using genetic positions from genome zipper. The 
genetic positions of these orthologs were extrapolated over the heatmap. Color of the heatmap illustrates the test-statistic of the Kruskal-wallis analysis. (PDF $35 \mathrm{~kb}$ )

Additional file 6: Figure S6. Phylogenetic tree of candidate heading genes PHYA, PHYB and PHYC. The evolutionary history was inferred by using the Maximum Likelihood method based on the JTT matrix-based model [67]. The tree is mid-point rooted, drawn to scale, with branch lengths proportional to the number of substitutions per site. All positions containing gaps and missing data were eliminated. Evolutionary analyses were conducted in MEGA 6.06 [66]. All the associated Lolium and Arabidopsis proteins were highlighted. (PDF $59 \mathrm{~kb}$ )

Additional file 7: Figure S7. Phylogenetic tree of candidate heading gene CRY2. The evolutionary history was inferred by using the Maximum Likelihood method based on the JTT matrix-based model [67]. The tree is mid-point rooted, drawn to scale, with branch lengths proportional to the number of substitutions per site. All positions containing gaps and missing data were eliminated. Evolutionary analyses were conducted in MEGA 6.06 [66]. All the associated Lolium and Arabidopsis proteins were highlighted. (PDF $40 \mathrm{~kb}$ )

Additional file 8: Figure S8. Phylogenetic tree of candidate heading gene PRR37. The evolutionary history was inferred by using the Maximum Likelihood method based on the JTT matrix-based model [67]. The tree is mid-point rooted, drawn to scale, with branch lengths proportional to the number of substitutions per site. All positions containing gaps and missing data were eliminated. Evolutionary analyses were conducted in MEGA 6.06 [66]. All the associated Lolium and rice proteins were highlighted. (PDF $38 \mathrm{~kb}$ )

Additional file 9: Figure S9. Phylogenetic tree of candidate heading gene SOC1. The evolutionary history was inferred by using the Maximum Likelihood method based on the JTT matrix-based model [67]. The tree is mid-point rooted, drawn to scale, with branch lengths proportional to the number of substitutions per site. All positions containing gaps and missing data were eliminated. Evolutionary analyses were conducted in MEGA 6.06 [66]. All the associated Lolium and Arabidopsis proteins were highlighted. (PDF $42 \mathrm{~kb}$ )

Additional file 10: Figure S10. Phylogenetic tree of candidate heading gene $\mathrm{CO}$. The evolutionary history was inferred by using the Maximum Likelihood method based on the JTT matrix-based model [67]. The tree is mid-point rooted, drawn to scale, with branch lengths proportional to the number of substitutions per site. All positions containing gaps and missing data were eliminated. Evolutionary analyses were conducted in MEGA 6.06 [66]. Associated Lolium and rice proteins were highlighted. (PDF $37 \mathrm{~kb}$ )

Additional file 11: Figure S11. Phylogenetic tree of candidate heading gene GI. The evolutionary history was inferred by using the Maximum Likelihood method based on the JTT matrix-based model [67]. The tree is mid-point rooted, drawn to scale, with branch lengths proportional to the number of substitutions per site. All positions containing gaps and missing data were eliminated. Evolutionary analyses were conducted in MEGA 6.06 [66]. Associated Lolium and Arabidopsis proteins were highlighted. (PDF $37 \mathrm{~kb}$ )

Additional file 12: Figure S12. Phylogenetic tree of candidate heading gene VRN1. The evolutionary history was inferred by using the Maximum Likelihood method based on the JTT matrix-based model [67]. The tree is mid-point rooted, drawn to scale, with branch lengths proportional to the number of substitutions per site. All positions containing gaps and missing data were eliminated. Evolutionary analyses were conducted in MEGA 6.06 [66]. Associated Lolium and barley proteins were highlighted. (PDF $40 \mathrm{~kb}$ )

Additional file 13: Data set S1. SNP positions and genotype calls. This additional data set contains SNP positions relative to the published draft genome [30]. The first column identifies the scaffold containing the SNP. The value after the final underscore identifies the SNP position within this scaffold. The second column identifies the alleles, and the remaining columns are the genotype calls for each individual. NN refers to a missing genotype. (ZIP 6379 kb)

\section{Abbreviations}

GWAS, genome wide association study; QTL, quantitative trait loci; LD, linkage disequilibrium; $L G$, linkage group

\section{Funding}

SKA is supported by a Teagasc PhD Walsh Fellowship. SLB is supported by an EU Marie-Sklodowska-Curie Fellowship (H2020-MSCA-IF: 658031). The study was funded through a DAFM project (RSF 14/S/819) and Teagasc core funding. The funders played no role in design of the study, collection of the data, analysis, and interpretation of data and in writing the manuscript.

\section{Availability of data and materials}

The datasets supporting the conclusions of this article are included within the article and its additional files.

\section{Authors' contributions}

DM, PC, SB, TRH conceived and designed the study. SKA, JV and SLB performed the data analysis. SKA, and SLB drafted the initial paper. SKA, SLB, DM, PC, JV, $\mathrm{TRH}$, and $\mathrm{SB}$ contributed to interpretation of data and preparation of the final manuscript. All authors read and approved the final version.

\section{Competing interests}

The authors declare that they have no competing interests.

\section{Consent for publication}

Not applicable.

\section{Ethics approval and consent to participate}

Not applicable.

\section{Author details}

${ }^{1}$ Teagasc, Crop Science Department, Oak Park, Carlow, Ireland. ${ }^{2}$ Teagasc, Grassland Science Research Department, Animal and Grassland Research and Innovation Centre, Oak Park, Carlow, Ireland. ${ }^{3}$ Department of Botany, Trinity College Dublin, Dublin 2, Dublin, Ireland.

Received: 27 April 2016 Accepted: 1 July 2016

Published online: 16 July 2016

\section{References}

1. McGrath S, Hodkinson TR, Barth S. Extremely high cytoplasmic diversity in natural and breeding populations of Lolium (Poaceae). Heredity. 2007;99(5):531-44.

2. Wilken D. Lolium. The Jepson Manual. Higher Plants of California, University of California Press, Berkeley. 1993; p. 1400.

3. Gagic M, Faville M, Kardailsky I, Putterill J. Comparative genomics and functional characterisation of the gigantea gene from the temperate forage perennial ryegrass Lolium perenne. Plant Mol Biol Rep. 2015;33(4): 1098-106.

4. Fischer G, Prieler S, van Velthuizen $H$, Berndes G, Faaij A, Londo M, de Wit M. Biofuel production potentials in europe: Sustainable use of cultivated land and pastures, part ii: Land use scenarios. Biomass Bioenergy. 2010;34(2):173-87.

5. O'Donoghue C, Creamer R, Crosson P, Curran T, Donnellan T, Farrelly N, Fealy R, French P, Geoghegan C, Green S, et al. Drivers of agricultural land use change in Ireland to 2025. Teagasc; 2015. http://citeseerx.ist.psu. edu/viewdoc/download?doi=10.1.1.704.6065\&rep=rep1\&type=pdf.

6. McGrath S, Hodkinson TR, Charles T, Zen D, Barth S. Variation in inflorescence characters and inflorescence development in ecotypes and cultivars of Lolium perenne L. Grass Forage Sci. 2010;65(4):398-409.

7. Smit H, Tas B, Taweel H, Tamminga S, Elgersma A. Effects of perennial ryegrass Lolium perenne L. cultivars on herbage production, nutritional quality and herbage intake of grazing dairy cows. Grass Forage Sci. 2005;60(3):297-309.

8. Humphreys MW, Yadav R, Cairns AJ, Turner L, Humphreys J, Skøt L. A changing climate for grassland research. New Phytologist. 2006;169(1): 9-26.

9. Yamada T, Jones E, Cogan N, Vecchies A, Nomura T, Hisano H, Shimamoto Y, Smith K, Hayward M, Forster J. Qtl analysis of morphological, developmental, and winter hardiness-associated traits in perennial ryegrass. Crop Sci. 2004;44(3):925-35.

10. Valk P, Proveniers M, Pertijs J, Lamers J, Dun C, Smeekens J. Late heading of perennial ryegrass caused by introducing an Arabidopsis homeobox gene. Plant Breed. 2004;123(6):531-5. 
11. Wilkins $P$. Breeding perennial ryegrass for agriculture. Euphytica. 1991;52(3):201-14.

12. Devos KM, Gale MD. Comparative genetics in the grasses. Plant Mol Biol. 1997;35(1-2):3-15.

13. Casler M, Vogel K. Accomplishments and impact from breeding for increased forage nutritional value. Crop Sci. 1999;39(1):12-20.

14. Frank A, Hofmann L. Light quality and stem numbers in cool-season forage grasses. Crop Sci. 1994;34(2):468-73.

15. Byrne S, Guiney E, Barth S, Donnison I, Mur LA, Milbourne D. Identification of coincident qtl for days to heading, spike length and spikelets per spike in Lolium perenne L. Euphytica. 2009;166(1):61-70.

16. Armstead IP, Turner LB, Farrell M, Skøt L, Gomez P, Montoya T, Donnison IS, King IP, Humphreys MO. Synteny between a major heading-date qtl in perennial ryegrass (Lolium perenne L.) and the hd3 heading-date locus in rice. Theor Appl Genet. 2004;108(5):822-8.

17. Jensen LB, Andersen JR, Frei U, Xing Y, Taylor C, Holm PB, Lübberstedt T. Qtl mapping of vernalization response in perennial ryegrass (Lolium perenne L.) reveals co-location with an orthologue of wheat vrn1. Theor Appl Genet. 2005;110(3):527-36

18. Armstead I, Turner L, Marshall A, Humphreys M, King I, Thorogood D. Identifying genetic components controlling fertility in the outcrossing grass species perennial ryegrass (Lolium perenne) by quantitative trait loci analysis and comparative genetics. New Phytologist. 2008;178(3):559-71.

19. Barre P, Moreau L, Mi F, Turner L, Gastal F, Julier B, Ghesquière M. Quantitative trait loci for leaf length in perennial ryegrass (Lolium perenne L.) Grass Forage Sci. 2009;64(3):310-21.

20. Studer B, Jensen LB, Hentrup S, Brazauskas G, Kölliker R, Lübberstedt T. Genetic characterisation of seed yield and fertility traits in perennial ryegrass (Lolium perenne L.) Theor Appl Genet. 2008;117(5):781-91.

21. Skøt L, Humphreys MO, Armstead I, Heywood S, Skøt KP, Sanderson R, Thomas ID, Chorlton KH, Hamilton NRS. An association mapping approach to identify flowering time genes in natural populations of Lolium perenne (L.) Mol Breed. 2005;15(3):233-45.

22. Skøt L, Humphreys J, Humphreys MO, Thorogood D, Gallagher J, Sanderson R, Armstead IP, Thomas ID. Association of candidate genes with flowering time and water-soluble carbohydrate content in Lolium perenne (L.) Genetics. 2007;177(1):535-47.

23. Shinozuka H, Cogan NO, Spangenberg GC, Forster JW. Quantitative trait locus (qtl) meta-analysis and comparative genomics for candidate gene prediction in perennial ryegrass (Lolium perenne L.) BMC Genetics. 2012;13(1):1.

24. Andersen JR, Jensen LB, Asp T, Lübberstedt T. Vernalization response in perennial ryegrass (Lolium perenne L.) involves orthologues of diploid wheat (Triticum monococcum) vrn1 and rice (Oryza sativa) hd1. Plant Mol Biol. 2006;60(4):481-94.

25. Fè D, Cericola F, Byrne S, Lenk I, Ashraf BH, Pedersen MG, Roulund N, Asp T, Janss L, Jensen CS, et al. Genomic dissection and prediction of heading date in perennial ryegrass. BMC Genomics. 2015;16(1):1.

26. Pfeifer M, Martis M, Asp T, Mayer KF, Lübberstedt T, Byrne S, Frei U, Studer B. The perennial ryegrass genomezipper: targeted use of genome resources for comparative grass genomics. Plant Physiol. 2013;161(2): 571-82.

27. Studer B, Byrne S, Nielsen RO, Panitz F, Bendixen C, Islam MS, Pfeifer M, Lübberstedt T, Asp T. A transcriptome map of perennial ryegrass (Lolium perenne L.) BMC Genomics. 2012;13(1):140.

28. Bates D, Maechler M, Bolker B, Walker S. Fitting Linear Mixed-Effects Models Using Ime4. Journal of Statistical Software. 67(1):1-48. doi:10.18637/jss.v067.i01.

29. Fè D, Pedersen MG, Jensen CS, Jensen J. Genetic and environmental variation in a commercial breeding program of perennial ryegrass. Crop Sci. 2015;55(2):631-40.

30. Byrne SL, Nagy I, Pfeifer M, Armstead I, Swain S, Studer B, Mayer K, Campbell JD, Czaban A, Hentrup S, et al. A synteny-based draft genome sequence of the forage grass Lolium perenne. Plant J. 2015;84(4):816-26.

31. Andrés F, Coupland $G$. The genetic basis of flowering responses to seasonal cues. Nat Rev Genet. 2012;13(9):627-39.

32. Kaul S, Koo HL, Jenkins J, Rizzo M, Rooney T, Tallon LJ, Feldblyum T, Nierman W, Benito MI, Lin X, et al. Analysis of the genome sequence of the flowering plant Arabidopsis thaliana. Nature. 2000;408(6814):796-815.

33. Vogel JP, Garvin DF, Mockler TC, Schmutz J, Rokhsar D, Bevan MW, Barry K, Lucas S, Harmon-Smith M, Lail K, et al. Genome sequencing and analysis of the model grass Brachypodium distachyon. Nature. 2010;463(7282):763-8.

34. Consortium IBGS, et al. A physical, genetic and functional sequence assembly of the barley genome. Nature. 2012;491(7426):711-6.

35. Schnable PS, Ware D, Fulton RS, Stein JC, Wei F, Pasternak S, Liang C, Zhang J, Fulton L, Graves TA, et al. The b73 maize genome: complexity, diversity, and dynamics. Science. 2009;326(5956):1112-5.

36. Paterson AH, Bowers JE, Bruggmann R, Dubchak I, Grimwood J, Gundlach H, Haberer G, Hellsten U, Mitros T, Poliakov A, et al. The Sorghum bicolor genome and the diversification of grasses. Nature. 2009;457(7229):551-6.

37. Project IRGS, et al. The map-based sequence of the rice genome. Nature. 2005;436(7052):793-800.

38. Gabaldón T. Large-scale assignment of orthology: back to phylogenetics. Genome Biol. 2008;9(10):235.

39. Mishra P, Panigrahi KC. Gigantea-an emerging story. Front Plant Sci. 2015;6:8.

40. Loukoianov A, Yan L, Blechl A, Sanchez A, Dubcovsky J. Regulation of vrn-1 vernalization genes in normal and transgenic polyploid wheat. Plant Physiol. 2005;138(4):2364-373.

41. Karlgren A, Gyllenstrand N, Källman T, Sundström JF, Moore D, Lascoux M, Lagercrantz U. Evolution of the pebp gene family in plants: functional diversification in seed plant evolution. Plant Physiol. 2011;156(4):1967-77.

42. Kobayashi Y, Kaya H, Goto K, Iwabuchi M, Araki T. A pair of related genes with antagonistic roles in mediating flowering signals. Science. 1999;286(5446):1960-62.

43. Peng FY, Hu Z, Yang RC. Genome-wide comparative analysis of flowering-related genes in Arabidopsis, wheat, and barley. Int J Plant Genomics. 2015;2015.

44. Kinoshita T, Ono N, Hayashi Y, Morimoto S, Nakamura S, Soda M, Kato Y, Ohnishi M, Nakano T, Inoue S-l, et al. Flowering locus t regulates stomatal opening. Curr Biol. 2011;21(14):1232-8.

45. Wang R, Albani MC, Vincent C, Bergonzi S, Luan M, Bai Y, Kiefer C, Castillo R, Coupland G. Aa tfl1 confers an age-dependent response to vernalization in perennial Arabis alpina. Plant Cell. 2011;23(4):1307-21.

46. Yamaguchi A, Kobayashi Y, Goto K, Abe M, Araki T. Twin sister of ft (tsf) acts as a floral pathway integrator redundantly with ft. Plant Cell Physiol. 2005;46(8):1175-89.

47. Yoo SJ, Chung KS, Jung SH, Yoo SY, Lee JS, Ahn JH. Brother of $\mathrm{ft}$ and $\mathrm{tfl}$ (bft) has tfl1-like activity and functions redundantly with tfl1 in inflorescence meristem development in Arabidopsis. Plant J. 2010;63(2): 241-53.

48. Jensen CS, Salchert K, Nielsen KK. A terminal flower1-like gene from perennial ryegrass involved in floral transition and axillary meristem identity. Plant Physiol. 2001;125(3):1517-28.

49. Williamson ML. Differential responses of tillers to floral induction in perennial ryegrass (Lolium perenne L.) : implications for perenniality. Massey University. Palmerston North, New Zealand. 2008. http://mro. massey.ac.nz/handle/10179/842.

50. Iwata H, Gaston A, Remay A, Thouroude T, Jeauffre J, Kawamura K, Oyant LH-S, Araki T, Denoyes B, Foucher F. The tfl1 homologue ksn is a regulator of continuous flowering in rose and strawberry. Plant J. 2012;69(1):116-25.

51. Elshire RJ, Glaubitz JC, Sun Q, Poland JA, Kawamoto K, Buckler ES, Mitchell SE. A robust, simple genotyping-by-sequencing (gbs) approach for high diversity species. PLOS ONE. 2011;6(5):19379.

52. Buffalo: Scythe - A Bayesian adapter trimmer version 0.994 BETA. 2011. https://github.com/vsbuffalo/scythe. Accessed 7 Nov 2015.

53. Joshi NA FJ. Sickle - A windowed adaptive trimming tool for FASTQ files using quality. 2011. https://github.com/ucdavis-bioinformatics/sickle. Accessed 7 Nov 2015.

54. Joshi NA FJ. Sabre - A barcode demultiplexing and trimming tool for FastQ files. 2011. https://github.com/najoshi/sabre. Accessed 7 Nov 2015

55. Li H, Durbin R. Fast and accurate short read alignment with burrows-wheeler transform. Bioinformatics. 2009;25(14):1754-60.

56. DePristo MA, Banks E, Poplin R, Garimella KV, Maguire JR, Hartl C, Philippakis AA, Del Angel G, Rivas MA, Hanna M, et al. A framework for variation discovery and genotyping using next-generation dna sequencing data. Nat Genet. 2011;43(5):491-8.

57. Lipka AE, Tian F, Wang Q, Peiffer J, Li M, Bradbury PJ, Gore MA, Buckler ES, Zhang Z. Gapit: genome association and prediction integrated tool. Bioinformatics. 2012;28(18):2397-9. 
58. Benjamini Y, Hochberg Y. Controlling the false discovery rate: a practical and powerful approach to multiple testing. J R Stat Soc Ser B (Methodological). 1995;57(1):289-300.

59. Bonferroni CE. Teoria statistica delle classi e calcolo delle probabilita. Pubblicazioni del R Istituto Superiore di Scienze Economiche e Commerciali di Firenze. 1936;8:3-62.

60. Chang CC, Chow CC, Tellier L, Vattikuti S, Purcell SM, Lee JJ. Second-generation plink: rising to the challenge of larger and richer datasets. Gigascience. 2015;4(1):1-16.

61. Gaunt TR, Rodríguez S, Day IN. Cubic exact solutions for the estimation of pairwise haplotype frequencies: implications for linkage disequilibrium analyses and a web tool'cubex'. BMC Bioinforma. 2007:8(1):428.

62. Team RC. A language and environment for statistical computing. Vienna, Austria. 2014; 2015.

63. UniProt: a hub for protein information. Nucleic acids research. 2015;43(Database issue):D204-12.

64. Proost $\mathrm{S}$, Van Bel $\mathrm{M}$, Vaneechoutte $\mathrm{D}$, Van de Peer $\mathrm{Y}$, Inzé $\mathrm{D}$, Mueller-Roeber B, Vandepoele K. Plaza 3.0: an access point for plant comparative genomics. Nucleic Acids Res. 2015;43(Database issue): D974-81.

65. Edgar RC. Muscle: multiple sequence alignment with high accuracy and high throughput. Nucleic Acids Res. 2004;32(5):1792-7.

66. Tamura K, Stecher G, Peterson D, Filipski A, Kumar S. Mega6: molecular evolutionary genetics analysis version 6.0. Mol Biol Evol. 2013;30(12): 2725-9.

67. Jones DT, Taylor WR, Thornton JM. The rapid generation of mutation data matrices from protein sequences. Comput Appl biosci CABIOS. 1992;8(3):275-82.

\section{Submit your next manuscript to BioMed Central and we will help you at every step:}

- We accept pre-submission inquiries

- Our selector tool helps you to find the most relevant journal

- We provide round the clock customer support

- Convenient online submission

- Thorough peer review

- Inclusion in PubMed and all major indexing services

- Maximum visibility for your research

Submit your manuscript at www.biomedcentral.com/submit 